\title{
DIGITIZATION PROGRAMS AT STScI
}

\author{
B.M. LASKER \\ Space Telescope Science Institute \\ Baltimore, $M D$ \\ U.S.A.
}

\section{General Remarks}

The Space Telescope Science Institute (STScI) continues with its programs of digitization, cataloging and data distribution. These were defined earlier, at the Geneva and the Edinburgh workshops (Jaschek 1989; MacGillivray \& Thomson 1992), and this report, by necessity of space, is incremental upon them.

The project to compress the scans used in the Guide Star Catalog (GSC; Lasker et al. 1990), as specified by White, Postman \& Lattanzi (1990), has advanced according to plan. The southern-hemisphere part of this compression program is now completed, and distribution arrangements for the CD ROM set will have been announced well before the publication of this volume. Similarly, the astrometric recalibration of the GSC has progressed as planned (Bucciarelli et al., this volume); and the development of photometric reference sequences (Postman et al. 1992) has continued in collaboration with a team from Torino (Ferrari et al., this volume).

It will be several years before a CD ROM distribution can be undertaken for the secondgeneration scans (see below). However, network access to small subsets of the data is practical, even now. As a pilot program, we have compressed a small number of plates, in the same format as developed for the CD ROM project (White, Postman \& Lattanzi 1990), archived them in the HST Archive (DMF/DADS; Long et al. 1993), and experimented with access procedures. After attention to a few technical and policy issues, we expect to implement this capability as a public facility in the Archive.

\section{Plate Scanning}

The scanning program, as presented earlier (Lasker 1992), consists of $15 \mu \mathrm{m}$ full-plate digitization of the approximately $3300 J, F$ and $N$ plates constituting the second Palomar Sky Survey and the second-epoch $F$ survey of the UK Schmidt. The program began in 1992 with the two PerkinElmer (PE) 2020G scanning microdensitometers nearly unchanged from the design configuration used for the GSC development (Lasker 1990). Throughput in that configuration was slow. Even so, about 200 scans have been made so far; and valuable experience has been gained with the plates and their properties. However, the completion of a program such as this in a reasonable 
time requires a scanning capability of approximately 1000 plates per year. Basing required microdensitometer modifications on this rate, we adopt a scanning time goal of 12 hours per plate, which corresponds, with time allocated for set-up, to a pixel rate of $60 \mu \mathrm{s}$.

\subsection{SERVO, DATA, AND CONTROL SYSTEMS}

The first upgrade implemented to achieve this rate reliably is the installation of modern servo, data, and control systems. These totally replace the original PE electronics, which while acceptable in the context of the initial design configuration and date, are limited by 1) a lack of external access to the servo, its frequency characteristics, or its error signal, 2) maintenance problems in the servo power area, and 3) a photometric data path tightly coupled to an archaic computer architecture.

The microdensitometers had already been equipped with metrology systems based on HP laser interferometers (Kinsey et al. 1984). Thus, it was a simple matter to upgrade the supporting electronics to a newer product, the HP model 5527A laser control system; this, configured with two HP model 10936A servo axis cards, closes the servo loops with microprocessor-controlled, user-tuned difference equations. One important detail is the generation of strobes to the photometric system as the scan crosses individual pixels. The timing of this signal, which has typical rates of tens of $\mathbf{k H z}$, is critical to the astrometric integrity of the system; and it must be generated electronically (i.e. firmware would not be fast enough). HP implemented this so-called 1/N circuitry for us as a custom item on a standard HP 10941A prototype card.

The analog signals from the servo controllers drive the original axes motors (Honeywell, now marketed by Pacific Scientific) by means of MultiTech TSD-series power amplifiers. These amplifiers are very efficient and reliable, and are capable of torquing the motors to the mechanical limits of the system. However, as they are of the switching type, extensive noise control measures were required to eliminate interference in the digital control signals and in the photometer (similar applications elsewhere might consider the alternative of quieter linear amplifiers).

The servos are interfaced to the host computer, in our case a VAXstation 3100 , by an IEEE-488 bus; and interfacing to the photometer and the miscellaneous digital control signals is done through a CAMAC system. The photometric data are organized for convenient and rapid block transfers to the host computer (typical transfer rates in the Mbyte $s^{-1}$ range) in CAMAC memory modules. We also note, for the benefit of others interested in similar microdensitometer modifications, that the IEEE-488 and CAMAC interfaces are supported on virtually all modern computers, and that CAMAC, while considered old in the physics-astronomy community, has retained a significant following in several industries and thus has good commercial support. In particular, the new servo system, being based largely on commercial components, may be an easy and economical upgrade for other PDS-type machines with laser metrology.

\subsection{LASER OPTICS AND MULTI-CHANNEL OPERATION}

The servo upgrades, while essential elements for reliability and precise control, by themselves do not overcome the basic speed restrictions of a single-channel scanning-type system. Accordingly, a multi-channel capability is essential to our goal of $12 \mathrm{hr}$ per plate. As the capability of the onechannel configuration is about $24 \mathrm{hr}$ per plate, a two or three channel configuration would meet our goals, while more channels would reach the faster domain in which we do have some interest.

A second design goal is to preserve, to the largest extent possible, the high photometric quality 
of the original optical system, which is based on the classical configuration of two microscopes, with discrete ingress and egress apertures to control scattered light. After reviewing a number of options, including configurations in use elsewhere, we adopted a HeNe $(6328 \AA)$ laser illuminated system, with the multi-channel capability being achieved by the use of an acoustooptic (AO) deflector. The scans then consist of full-length mechanical traverses across the plate in the $x$-direction, coordinated with displacements of a few channels by the AO deflector in the y-direction. The closest similar application is the Cambridge APM (Kibblewhite et al. 1984), but note also the Minnesota and Sacramento Peak systems (Pennington et al. 1993; Arrambide et al. 1984).

The most difficult theoretical design issue in a laser machine is photometric linearity, the underlying physical issue being that the coherent laser illumination has a wavelength comparable to the scale of the plate granularity. This has been analyzed extensively by Swing (1976). While the linearity conditions developed therein are not generally satisfied, experience with typical astronomical objects on standard emulsions at several institutions show that good practical results are obtained (cf. discussion in Lasker 1993).

The selection of scanning spot size is based on sampling theory. For our $15 \mu \mathrm{m}$ pixels, a spot large enough and smooth enough to have a falling response that becomes small (ideally zero) at the Nyquist frequency $\left(f_{N}=1 / 30 \mu \mathrm{m}^{-1}\right)$ is required. The Gaussian spot that is naturally formed by convergent optics in a mode 00 laser beam meets this criterion well. Optical elements are adopted such that the spot profile is exponential with $\sigma=10.6 \mu \mathrm{m}$, which corresponds to a full width of $30 \mu \mathrm{m}$ at $e^{-1}$ and $42 \mu \mathrm{m}$ at $e^{-2}$. The corresponding response at $f_{N}$ is 0.085 , which appears to be a good compromise; a larger beam would give less aliasing, but at the price of resolution.

On the practical side, minimization of the inevitable halo around a laser spot is critical to the photometric integrity of the system. As no defining aperture can be used on the egress side, a faint halo, e.g. passing through sky background while the beam is in the center of a nearly saturated star, will give a falsely low density reading for the pixels in the star. Steps taken to achieve a very clean laser spot include 1) the use of high quality surfaces, with optimized coatings whenever possible, in the spot forming optics, mirrors and platen, 2) a spatial filter to eliminate spurious laser modes from the beam, and 3) the avoidance of occulting edges within many radii of the beam. Additionally, a large aperture (about $250 \mu \mathrm{m}$ deprojected) is used in the egress optics. Measurements of the actual beam profile (slit scans in the real scanning environment) show a narrow profile that falls exponentially to a relative intensity of $10^{-3}$ at $63 \mu \mathrm{m}$ from the spot center and $10^{-4}$ at $126 \mu \mathrm{m}$ therefrom.

The acousto-optic deflector is a $\mathrm{TeO}_{2}$ crystal, cut in the slow-shear mode (Isomet LS-55V); and the deflection sensitivity is about $200 \mathrm{kHz}$ per $15 \mu \mathrm{m}$ pixel. The required channel-frequency correspondence in table-driven from a digital signal processor (DSP) which is synchronized to the $1 / N$ signals generated in the $x$-servo; and the radii-frequency excitation is generated by a SCITEQ ADS-432 synthesizer. The DSP also adds a function of the y-servo error to the table-generated frequencies, so as to remove any position error ('chopping') caused by servo imbalance.

The completed laser illumination system is shown in Fig. 1. Note that the optics are constructed on an optical shelf interchangeable with the original PE one. This permits us to return to the classical two-microscope configuration quickly, and thus to retain a capability for those scanning projects not well suited to laser illumination. The egress optics, which are not shown because of space limitations, are similar to the original, the major change being a dichroic to maximise the white light wide-field illumination to the viewing screen, while minimizing the intensity of the laser spot thereon. 


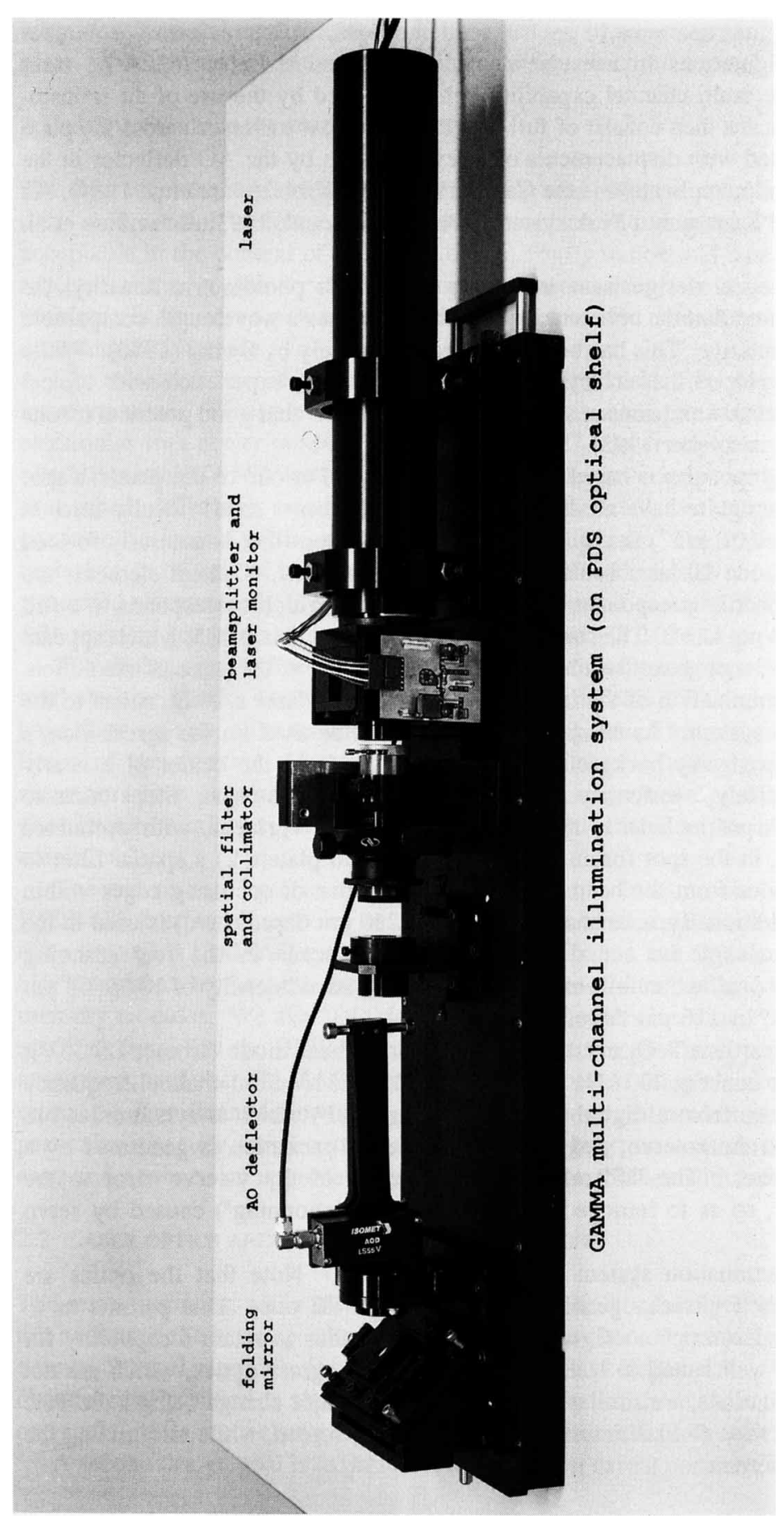

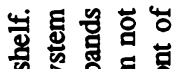
สํ.

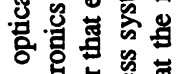
此

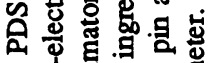

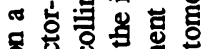
용 \& 8

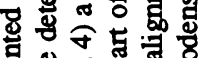

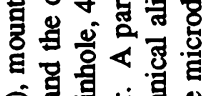

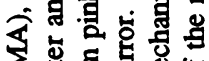

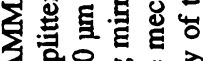

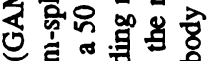
\& 형영 तु 要 更 들 8 둥

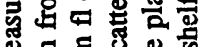

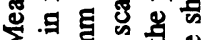

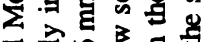
중

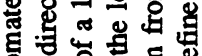
으응 是 일 क 5 \%

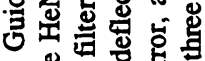

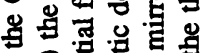
영

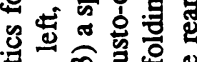
후응 筞

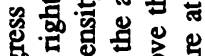

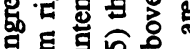

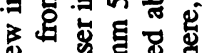

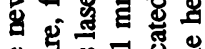
氙 政 和递

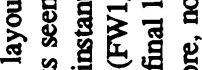
궁요

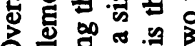
○등요

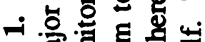

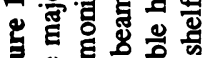

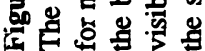




\section{Acknowledgements}

The compression program reported herein is conducted by Project Scientist Marc Postman, together with colleagues Jesse B. Doggett, Flavio J. Mendez, and Michael G. Meakes. Incorporation of compressed scans in the HST Archive is supported by Stefi A. Baum, Knox S. Long, Conrad R. Sturch, and colleagues. The engineering aspects of the GAMMA implementation are performed by Anatoly Evzerov, Gretchen R. Greene, Victoria G. Laidler, Knute A. Ray, and colleagues, together with advisor Marc Damashek. ST ScI is operatd by AURA, Inc., under contract to NASA.

\section{References}

Arrambide, M.R., Dunn, R.B., Healy, A.W., Porter, R., Widner, A.L., November, L.J. and Spence, G.E., 1984. In 'Astronomical Microdensitometry', ed. D.E. Klinglesmith, NASA CP2317, p. 277.

Jaschek, C., editor, 1989. 'Digitized Optical Sky Surveys', in Bull. Inform. du CDS, Number 37.

Kibblewhite, E.J., Bridgeland, M.T., Bunclark, P. and Irwin, M., 1984. In 'Astronomical Microdensitometry', ed. D.A. Klinglesmith, NASA CP-2317, p. 277.

Kinsey, J.H., Denman, R.J. and Evzerov, A., 1984. Rev. Sci. Instrum., 55, 1960.

Lasker, B.M., 1992. In 'Digitised Optical Sky Surveys', eds. H.T. MacGillivray and E.B. Thomson, Kluwer Academic Publishers, Dordrecht, p. 87.

Lasker, B.M., 1993. In 'Databases for Galactic Structure', ed. A.G.D. Phillip, in press.

Lasker, B.M., Sturch, C.R., McLean, B.J., Russell, J.L., Jenkner, H. and Shara, M., 1990. Astron. J., 89, 2019.

Long, K.S., Baum, S.A., Borne, K. and Swade, D., 1993. In 'Astron. Data Analysis Software and Systems. III.', eds. D. Crabtree, R.J. Hanisch and J. Barnes, A.S.P. Conf. Series, in press.

MacGillivray, H.T. and Thomson, E.B., editors, 1992. 'Digitised Optical Sky Surveys', Kluwer Academic Publishers, Dordrecht.

Pennington, R.L., Humphreys, R.M., Odewahn, S.C., Zumach, W. and Thurmes, P.M., 1993. Pub. Astr. Soc. Pacific, 105, 521.

Postman, M., Siciliano, L., Shara, M., Rehner, D., Brosch, N., Sturch, C., Bucciarelli, B. and Lopez, C., 1992. In 'Digitised Optical Sky Surveys', eds. H.T. MacGillivray and E.B. Thomson, Kluwer Academic Publishers, Dordrech,. p. 61.

Swing, R.E., 1976. Optical Engineering, 15, 559.

White, R.L., Postman, M. and Lattanzi, M.G., 1992. In 'Digitised Optical Sky Surveys', eds. H.T. MacGillivray and E.B. Thomson, Kluwer Academic Publishers, Dordrecht, p. 167. 